\title{
Some Aspects of Supersymmetric Field Theories with Minimal Length and Maximal Momentum
}

\author{
Kourosh Nozari, ${ }^{1}$ F. Moafi, ${ }^{2}$ and F. Rezaee Balef ${ }^{2}$ \\ ${ }^{1}$ Department of Physics, Faculty of Basic Sciences, University of Mazandaran, P.O. Box 47416-1467, Babolsar, Iran \\ ${ }^{2}$ Department of Physics, Islamic Azad University, Sari Branch, Sari, Iran
}

Correspondence should be addressed to Kourosh Nozari; knozari@umz.ac.ir

Received 21 May 2013; Accepted 17 July 2013

Academic Editor: Chao-Qiang Geng

Copyright (c) 2013 Kourosh Nozari et al. This is an open access article distributed under the Creative Commons Attribution License, which permits unrestricted use, distribution, and reproduction in any medium, provided the original work is properly cited.

We consider a real scalar field and a Majorana fermion field to construct a supersymmetric quantum theory of free fermion fields based on the deformed Heisenberg algebra $[x, p]=i \hbar\left(1-\beta p+2 \beta^{2} p^{2}\right)$, where $\beta$ is a deformation parameter. We present a deformed supersymmetric algebra in the presence of minimal length and maximal momentum.

\section{Introduction}

Physics in extremely high energy regions is particularly of interest to particle physicists. One of the promising idea in the realm of high energy physics is the idea of supersymmetry (SUSY). (See for instance [1] and references therein.) This is a symmetry that relates or mixes (unites) fermions and bosons. Supersymmetry has the potential to solve some outstanding mysteries in particle physics. One important problem that stands out is called the hierarchy problem. It is believed that the mass of the Higgs boson, $m_{h}$, is much smaller than the fundamental Planck mass, $m_{p}=\sqrt{\hbar c / G} \approx 10^{19} \mathrm{GeV} / c^{2}$. On the other hand, when we discuss gravity in the context of the quantum field theory, it is expected that there is a minimal measurable length that restricts resolution of adjacent spacetime points up to the Planck length. This is actually a common address of all quantum gravity candidates. (See [2] and references therein.) Incorporation of this idea in quantum field theory provides a theory that is naturally regularized in the ultraviolate regime [3-5]. Recently in the context of the doubly special relativity it has been revealed that a test particle's momentum cannot be arbitrarily imprecise leading nontrivially to the result that there is a maximal momentum for test particles [6-12]. This idea can be explained also through modified dispersion relations as a phenomenological outcome of foam-like structure of spacetime at the Planck scale $[13,14]$. String theory which has a characteristic scale $\sqrt{\alpha}$ is one of the most successful theoretical framework which overcomes the difficulty of ultraviolet divergence in quantum theory of gravity. Therefore, if we construct a field theory which captures some stringy nature and/or includes stringy corrections, it would play a pivotal role in investigating physics in high energy regions even near the Planck scale. One way to discuss these corrections is deforming the standard Heisenberg uncertainty principle to a generalized uncertainty principle (GUP). A GUP that predicts a minimal observable length can be written as follows:

$$
\Delta x \Delta p \geq \frac{\hbar}{2}+\beta_{0} e_{p l}^{2} \frac{(\Delta p)^{2}}{\hbar}
$$

This GUP leads to the following algebraic structure

$$
[x, p]=i \hbar\left(1+\beta^{2} p^{2}\right)
$$

where $\beta=\beta_{0} /\left(M_{p l} c\right)^{2}=\beta_{0} \ell_{p l}^{2} / \hbar^{2}$. If GUP is realized in a certain string theory context, $\beta$ takes a value of the order of the string scale, $\beta \sim \alpha$. In the presence of both minimal length and maximal momentum, the GUP can be given as follows:

$$
\Delta x \Delta p \geq \frac{\hbar}{2}\left(1-2 \beta\langle p\rangle+4 \beta^{2}\left\langle p^{2}\right\rangle\right) .
$$


In this framework the following algebraic structure can be deduced [15]:

$$
[x, p]=i \hbar\left(1-\beta p+2 \beta^{2} p^{2}\right) \text {. }
$$

In this context, we can define the generalized momentum operator as

$$
\mathbf{P}=p\left(1-\beta p+2 \beta^{2} p^{2}\right)
$$

With these preliminaries, in this paper we construct a quantum theory of free fermion fields based on the deformed Heisenberg algebra (4). We respect the supersymmetry in this context as a guiding principle. This is because string theory contains this symmetry, and it provides a useful tool to understand physics in ultraviolet regime. Because of existence of natural cutoffs as minimal length and maximal momentum, the supersymmetry is deformed in this context. From the fermionic part of the system, we propose an action of fermionic field based on the GUP in a spacetime with arbitrary number of dimensions. We construct the deformed supersymmetric algebra in this context. Finally, we compute some scattering amplitudes such as the pair annihilation amplitude to see how these important quantities can be calculated in the presence of natural cutoffs. We note that experimental limits and the general phenomenology perspective in this issue are not discussed here. One can see [16] for this purpose. We note also that, while the study of SUSY in relation to GUP is a relatively young subject, a bit more has been done for other quantum-spacetime intuitions. For instance, in [17] the authors have studied the basic twist quantization of $\operatorname{sep}(1 \mid 2)$ and kappa-deformation of $D=1$ superconformal mechanics.

The organization of the paper is as follows. In Section 2, we construct a scalar field theory based on the GUP (4). Section 3 is devoted to the issue of supersymmetry in the presence of natural cutoffs as minimal length and maximal momentum. In Section 4, we compute scattering amplitude of the pair annihilation in the presence of natural cutoffs. The paper follows by a summary and conclusion in Section 5.

\section{Scalar Field Theory}

Generalization of the Heisenberg algebra to $d$-dimensions, where rotational symmetry is preserved and there are both a minimal length and maximal momentum as natural cutoffs, is as follows:

$$
\left[\mathbf{x}_{i}, \mathbf{p}_{j}\right]=i \hbar\left(1-\beta \mathbf{p}+2 \beta^{2} \mathbf{p}^{2}\right) \delta_{i j}
$$

Here $i$ and $j$ run from 1 to $d$, where in three dimensions $\mathbf{p}=p_{x} \mathbf{i}+p_{y} \mathbf{j}+p_{z} \mathbf{k}$ where $\mathbf{i}, \mathbf{j}$, and $\mathbf{k}$ are unit vectors of cartesian coordinates and $\mathbf{p}^{2}=\sum_{i=1}^{d}\left(p_{i}\right)^{2}$. Hereafter, we use indices $i$ and $j$ for spatial coordinates and $a$ and $b$ for all spacetime coordinates. Now the Jacobi identity determines the full algebra of the theory which is given as follows (see the work by Nozari and Etemadi in [11]):

$$
\begin{gathered}
{\left[\mathbf{x}_{i}, \mathbf{p}_{j}\right]=i \hbar\left(1-\beta \mathbf{p}+2 \beta^{2} \mathbf{p}^{2}\right) \delta_{i j},} \\
{\left[\mathbf{p}_{i}, \mathbf{p}_{j}\right]=0} \\
{\left[\mathbf{x}_{i}, \mathbf{x}_{j}\right]=-i \beta \hbar\left(4 \beta-\frac{1}{\mathbf{p}}\right)\left(1-\beta \mathbf{p}+2 \beta^{2} \mathbf{p}^{2}\right) \mathbf{L}_{i j} .}
\end{gathered}
$$

The presence of the $1 / \mathbf{p}$ is a trace of the existence of the maximal momentum in this setup. Note also that (9) reflects the noncommutative nature of the spacetime manifold in Planck scale. Here $\mathbf{L}_{i j}$ are angular momentum like operators defined as

$$
\mathbf{L}_{i j}=\frac{1}{1-\beta \mathbf{p}+2 \beta^{2} \mathbf{p}^{2}}\left(\mathbf{x}_{i} \mathbf{p}_{j}-\mathbf{x}_{j} \mathbf{p}_{i}\right) .
$$

Since operators $\mathbf{p}^{i}$ s commute with each other, and we have not assumed the existence of a minimal uncertainty in momentum (see [18] for possible extension in this regard), we can construct our theory in momentum space representation. In momentum space representation, momentum operators are diagonalized simultaneously, and we do not distinguish eigenvalues of momentum $\mathbf{p}_{i}$ from operator $\mathbf{p}^{i}$. In what follows, we set $\hbar=1$ for simplicity. The Lagrangian in $d+1$ dimensional spacetime and in the presence of a minimal observable length and a maximal momentum can be written as follows:

$$
\begin{gathered}
\mathscr{L}=-\frac{1}{2} \int_{-p_{p l}}^{+p_{p l}} d^{d} p\left(1-\beta \mathbf{p}+2 \beta^{2} \mathbf{p}^{2}\right)^{-1} \Phi(-\mathbf{p}, t) \\
\times\left[\partial_{t}^{2}+\mathbf{p}^{2}+m^{2}\right] \Phi(\mathbf{p}, t),
\end{gathered}
$$

where

$$
\mathbf{p}^{2}=\sum_{i=1}^{d}\left(p_{i}\right)^{2}
$$

Note that the difference from ordinary quantum field theory is the extra prefactor $\left(1-\beta \mathbf{p}+2 \beta^{2} \mathbf{p}^{2}\right)^{-1}$ in the Lagrangian. Using the Bjorken-Johnson-Low prescription, from behavior of $T^{*}$-product through the following relations:

$$
\begin{aligned}
& \lim _{\mathbf{q} \rightarrow \infty}\left\langle T^{*} \widehat{\Phi}(\mathbf{p}, \mathbf{q}) \widehat{\Phi}\left(\mathbf{p}^{\prime}, \mathbf{q}^{\prime}\right)\right\rangle, \\
& \lim _{\mathbf{q} \rightarrow \infty} \mathbf{q}\left\langle T^{*} \widehat{\Phi}(\mathbf{p}, \mathbf{q}) \widehat{\Phi}\left(\mathbf{p}^{\prime}, \mathbf{q}^{\prime}\right)\right\rangle,
\end{aligned}
$$

where

$$
T^{*} \widehat{\Phi}(\mathbf{p}, \mathbf{q}) \widehat{\Phi}\left(\mathbf{p}^{\prime}, \mathbf{q}^{\prime}\right)=T \widehat{\Phi}(\mathbf{p}, \mathbf{q}) \widehat{\Phi}\left(\mathbf{p}^{\prime}, \mathbf{q}^{\prime}\right)
$$

we obtain

$$
\left[\widehat{\Phi}(\mathbf{p}, t), \dot{\widehat{\Phi}}\left(\mathbf{p}^{\prime}, t\right)\right]=i \hbar\left(1-\beta \mathbf{p}+2 \beta^{2} \mathbf{p}^{2}\right) \delta\left(\mathbf{p}+\mathbf{p}^{\prime}\right),
$$


where

$$
\begin{aligned}
\widehat{\Phi}(\mathbf{p}, t)= & \frac{\hbar}{E(\mathbf{p})} \widehat{\phi}(\mathbf{p}) \exp \left(\frac{t}{i \hbar} E(\mathbf{p})\right) \\
& +\frac{\hbar}{E(-\mathbf{p})} \widehat{\phi}^{\dagger}(-\mathbf{p}) \exp \left(\frac{-t}{i \hbar} E(-\mathbf{p})\right) .
\end{aligned}
$$

In this relation, $E(\mathbf{p}) \equiv \sqrt{Z(\mathbf{p})+m^{2}}$ where $Z(\mathbf{p})$ is an arbitrary even function whose explicit form has no influence on the arguments. One can see from (15) that a deformation prefactor, $\left(1-\beta \mathbf{p}+2 \beta^{2} \mathbf{p}^{2}\right)$, of Heisenberg algebra in the first quantization (6) also appears in canonical commutation relation of the second quantized field theory.

In the fermion field case, we encounter a difficulty in the construction of the second quantized Hilbert space which does not appear in a scalar field system. In what follows we use the idea of supersymmetry to construct a quantized field theory of fermions. In fact, we construct a quantum field theory of fermions, which is consistent with the above scalar field theory, and uses the supersymmetry prescription with a minimal length and maximal momentum scales.

\section{Supersymmetry and GUP}

Supersymmetric quantum field theory with minimal length has been studied by Shibusa [19]. In what follows, we generalize the pioneer work of Shibusa to the more general case that there are both minimal length and maximal momentum as natural cutoffs.

As we have mentioned in Section 1, supersymmetry proposes that to each fermion there exists a boson and vice versa. Thus in two- and three-dimensional spacetime, a system with a real scalar and a Majorana fermion has a special symmetry between a boson and a fermion, namely, SUSY. Following [19], our notation for two- and three-dimensional spacetime is as follows: in those dimensional spacetime (with signature $(-,+)$ or $(-,+,+))$ the Lorantz group has a real (Majorana) two-component spinor representation $\psi^{\alpha}$. For instance, in three-dimensional spacetime, we define a representation of Gamma matrices by Pauli matrices as follows [19]:

$$
\begin{gathered}
\left\{\Gamma^{a}, \Gamma^{b}\right\}=2 \eta^{a b}=2 \operatorname{diag}(-++), \\
\Gamma^{0}=-i \sigma_{2}=i \sigma^{2}, \quad \Gamma^{1}=\sigma_{1}=-\sigma^{1}, \quad \Gamma^{2}=-\sigma_{3} .
\end{gathered}
$$

We note that the spinor indices in this case are lowered or raised by charge conjugation matrix $C_{\alpha \beta} \equiv \Gamma^{0}$ and its inverse matrix $C^{-1}$. The generalized supersymmetric algebra in the presence of a minimal length and a maximal momentum and its action on a scalar field $\phi$, a Majorana fermion $\psi$, and an auxiliary field $F$ with parameter $\epsilon^{\alpha}$ is as follows:

$$
\begin{aligned}
{\left[\bar{\epsilon}_{1} Q, \bar{\epsilon}_{2} Q\right] } & =2 \Upsilon \bar{\epsilon}_{1} \Gamma^{a} \epsilon_{2} p_{a}, \\
\delta \phi(p, t) & =i \bar{\epsilon} \psi(p, t),
\end{aligned}
$$

$$
\begin{aligned}
\delta \psi^{\alpha}(p, t)= & \lambda_{1} F(p, t) \epsilon^{\alpha} \\
& -\lambda_{2}\left\{\left(\bar{\epsilon} \Gamma^{0} C^{-1}\right)^{\alpha} \partial_{t}+\left(\bar{\epsilon} \Gamma^{j} C^{-1}\right)^{\alpha}\left(i p_{j}\right)\right\} \phi(p, t),
\end{aligned}
$$

$$
\delta F(p, t)=\lambda_{3} i \bar{\epsilon}\left[\Gamma^{0} \partial_{t}+\Gamma^{j}\left(i p_{j}\right)\right] \psi(p, t),
$$

where $\Upsilon$ and $\lambda_{i}$ are functions of GUP deformation parameter $\beta$ and momentum. These factors reduce to unity in the limit of $\beta \rightarrow 0$ and will be determined later by consistency condition. The closeness of algebra requires

$$
\lambda_{1} \lambda_{3}=\lambda_{2}=\Upsilon \text {. }
$$

Now the supersymmetric Lagrangian can be written as a sum of separate Lagrangians; that is,

$$
\mathscr{L}=\mathscr{L}_{B}+\mathscr{L}_{F}+\mathscr{L}_{\text {aux }},
$$

where $\mathscr{L}_{B}, \mathscr{L}_{F}$, and $\mathscr{L}_{\text {aux }}$ are Bosonic, Fermionic, and auxiliary fields Lagrangian, respectively. Following [19], we generalize the Lagrangian by introduction of factors $\zeta_{i}$, which are functions of deforming parameter $\beta$ and momentum. These functions can be determined as follows. Starting with Lagrangian,

$$
\begin{aligned}
& \mathscr{L} \\
& \begin{aligned}
=-\frac{1}{2} \int_{-p_{p l}}^{+p_{p l} l} d p^{d}\left\{\zeta_{1} \phi(-p, t)\left(\partial_{t}^{2}+\mathbf{p}^{2}+m^{2}\right) \phi(p, t)\right. \\
+i \zeta_{2} \bar{\psi}(-p, t)\left(\Gamma^{0} \partial_{t}+\left(i p_{i}\right) \Gamma^{i}+m\right) \psi(p, t) \\
+\sqrt{\zeta_{1} \zeta_{3}} m \phi(-p, t) F(p, t) \\
\left.-\zeta_{3} F(-p, t) F(p, t)\right\},
\end{aligned}
\end{aligned}
$$

where $d$ is the number of the spatial coordinates. By integrating out the field $F$, we obtain the Lagrangian for the scalar and Majorana fields as follows:

$$
\begin{aligned}
\mathscr{L}=-\frac{1}{2} \int_{-p_{p l}}^{+p_{p l}} d p^{d}\{ & \left\{\zeta_{1} \phi(-p, t)\left(\partial_{t}^{2}+\mathbf{p}^{2}+m^{2}\right) \phi(p, t)\right. \\
& +i \zeta_{2} \bar{\psi}(-p, t) \\
& \left.\times\left(\Gamma^{0} \partial_{t}+\left(i p_{i}\right) \Gamma^{i}+m\right) \psi(p, t)\right\} .
\end{aligned}
$$

On the other hand, the invariance of the Lagrangian (24) under supersymmetry variation (19)-(21) in the presence of the minimal length and maximal momentum leads to

$$
\begin{gathered}
\lambda_{1} \zeta_{2}=\sqrt{\zeta_{1} \zeta_{3}}, \\
\lambda_{1} \zeta_{2}=\lambda_{3} \zeta_{3}, \\
\zeta_{1}=\lambda_{1} \lambda_{3} \zeta_{2} .
\end{gathered}
$$


Using the conditions (22) and (26), only $\zeta_{1}$ and $\zeta_{2}$ need to be determined. Note also that factor $\lambda_{1}$ can be absorbed into normalization of $F$. In what follows, we set $\lambda_{1}=1$ for $F$ to be an auxiliary field. Noether's current for supersymmetric Lagrangian (25) can be calculated, and the supersymmetric charge is obtained to be

$$
\begin{aligned}
& Q^{\alpha} \\
& =\int d t \int_{-p_{p l}}^{+p_{p l}} d p^{d} \zeta_{1}\left\{-\psi^{\alpha}(-p, t) \partial_{t} \phi(p, t)+\left(\Gamma^{i} \Gamma^{0} \psi(-p, t)\right)^{\alpha}\right. \\
& \times\left(i p_{i}\right) \phi(p, t) \\
& \left.+m\left(\Gamma^{0} \psi(-p, t)\right)^{\alpha} \phi(p, t)\right\} .
\end{aligned}
$$

Now the Hamiltonian of this system can be written as follows:

$$
\mathscr{H}=p^{0}=-\frac{1}{4} \frac{\zeta_{2}}{\zeta_{1}}\left(C \Gamma^{0}\right)_{\alpha \beta}\left\{Q^{\alpha}, Q^{\beta}\right\} .
$$

Using the Bjorken-Johnson-Low prescription, from behaviors of $T^{*}$-product between fields, as has been explained in the previous section, we obtain canonical relations as follows:

$$
\begin{gathered}
{\left[\phi(p, t), \partial_{t} \phi(q, t)\right]=i \zeta_{1}^{-1} \delta(p+q),} \\
\left\{\psi^{\alpha}(p, t), \psi^{\beta}(q, t)\right\}=-\zeta_{2}^{-1}\left(\Gamma^{0} C^{-1}\right)^{\alpha \beta} \delta(p+q) .
\end{gathered}
$$

Therefore, the Hamiltonian (28) now can be written as follows:

$$
\begin{aligned}
\mathscr{H}=\frac{1}{2} \int_{-p_{p l}}^{+p_{p l}} d p^{d} \zeta_{1}\{ & \pi(-p, t) \pi(p, t) \\
& \left.+\phi(-p, t)\left(\mathbf{p}^{2}+m^{2}\right) \phi(p, t)\right\} \\
& +i \zeta_{2} \bar{\psi}(-p, t)\left(\left(i p_{i}\right) \Gamma^{i}+m\right) \psi(p, t),
\end{aligned}
$$

where $\pi(p, t) \equiv \partial_{t} \phi(-p, t)$ and $i=1,2, \ldots, d$. There is another condition that can be used to determine $\zeta_{1}$ and $\zeta_{2}$ and comes from the free energy of the supersymmetric vacuum. From algebra (18), the supersymmetric state has zero energy:

$$
\begin{aligned}
0= & \frac{1}{2} \operatorname{Tr}_{B} \ln \left[\zeta_{1}\left(E^{2}+\mathbf{p}^{2}+m^{2}\right)\right] \\
& -\frac{1}{4} \operatorname{Tr}_{F} \ln \left[\zeta_{1}\left(E^{2}+\mathbf{p}^{2}+m^{2}\right)\right],
\end{aligned}
$$

where $\operatorname{Tr}_{B}$ and $\operatorname{Tr}_{F}$ represent trace in bosonic and fermionic Hilbert space, respectively. This relation leads to the following condition:

$$
\zeta_{1}=\zeta_{2}^{2}
$$

Finally we can write $\zeta_{1}=\left(1-\beta \mathbf{p}+2 \beta^{2} \mathbf{p}^{2}\right)^{-1}$ as one can read from (11). Thus we have

$$
\begin{gathered}
\Upsilon=\lambda_{2}=\lambda_{3}=\zeta_{2}=\left(1-\beta \mathbf{p}+2 \beta^{2} \mathbf{p}^{2}\right)^{-1 / 2}, \\
\lambda_{1}=\zeta_{3}=1, \\
\zeta_{1}=\left(1-\beta \mathbf{p}+2 \beta^{2} \mathbf{p}^{2}\right)^{-1} .
\end{gathered}
$$

Therefore, we have constructed the quantized field of fermions as follows:

$$
\begin{aligned}
& \left\{\psi^{\alpha}(p, t), \psi^{\beta}(q, t)\right\} \\
& \quad=-\left(1-\beta \mathbf{p}+2 \beta^{2} \mathbf{p}^{2}\right)^{1 / 2}\left(\Gamma^{0} C^{-1}\right)^{\alpha \beta} \delta(p+q) ;
\end{aligned}
$$

in consequence, the supersymmetric algebra in the presence of both minimal length and maximal momentum is deformed from the usual one as follows:

$$
\left[\bar{\epsilon}_{1} Q, \bar{\epsilon}_{2} Q\right]=2\left(1-\beta \mathbf{p}+2 \beta^{2} \mathbf{p}^{2}\right)^{-1 / 2} \bar{\epsilon}_{1} \Gamma^{a} \epsilon_{2} p_{a} .
$$

These results can be generalized to higher dimensions easily. Finally we note that the Lagrangian now takes the following form:

$$
\begin{aligned}
\mathscr{L}=\int_{-p_{p l}}^{+p_{p l}} d^{d} p\{ & -i\left(1-\beta \mathbf{p}+2 \beta^{2} \mathbf{p}^{2}\right)^{-1 / 2} \bar{\psi}(-p, t) \\
& \left.\times\left[\Gamma^{0} \partial_{t}+\left(i p_{i}\right) \Gamma^{i}+m\right] \psi(p, t)\right\} .
\end{aligned}
$$

We see that in the presence of quantum gravity effects (as minimal length and maximal momentum) there is an extra, universal prefactor $\left(1-\beta \mathbf{p}+2 \beta^{2} \mathbf{p}^{2}\right)^{-1 / 2}$ in comparison with the usual fermion action, regardless of existence or absence of supersymmetry. We note that the major difference between our framework and the formalism presented in [19] is the difference in the mentioned prefactor. Our prefactor contains both minimal length and maximal momentum simultaneously. Otherwise, the algebraic structure of the two supersymmetric algebras is the same.

\section{Some Scattering Amplitudes in the Presence of Minimal Length and Maximal Momentum}

In a perturbative expansion one usually refines the calculations using corrections that are becoming smaller and smaller. The reduction in importance is quantified by the power of the perturbation parameter, which is usually the coupling strength. At some point we are adding refinements that are too small to be measured, and we know we can stop adding refinements. In a perturbation expansion, the amplitude $M$ for a given process can be computed using an expansion of the type [20]

$$
M=\sum_{n} g^{k_{n}} M_{n}
$$

where $g^{k_{n}}$ is the coupling constant. In what follows we are going to see the effect of minimal length and maximal momentum as natural cutoffs on the scattering amplitude of the annihilation-creation process with spin-0 boson. In the presence of just a minimal measurable length as ultraviolet cutoff, momentum generalizes to $p \rightarrow p\left(1+\beta p^{2}\right)$. So we have

$$
M=\int_{-\infty}^{+\infty} \frac{-i g^{2} d p}{\left(1+\beta p^{2}\right)\left[p^{2}\left(1+\beta p^{2}\right)^{2}-m_{B}^{2}\right]}
$$




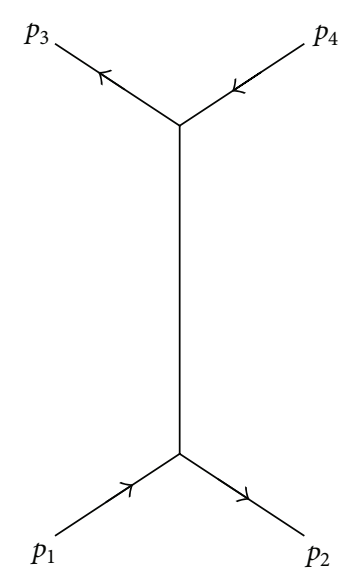

FIgURE 1: The geometry of the annihilation-creation process.

We have discarded the remaining delta function which is

$$
(2 \pi)^{4} \delta\left(p_{1}+p_{2}-p_{3}-p_{4}\right)
$$

where $p_{i}$ are defined in the geometry of the process as shown in Figure 1.

Simplifying relation (38), we find

$$
M=\int_{-\infty}^{+\infty} \frac{-i g^{2} d p}{p\left(1+\beta p^{2}\right)^{3}-m_{B}\left(1+\beta p^{2}\right)^{2}} .
$$

By solving the integral, we obtain

$$
\begin{array}{r}
M=-i g^{2}\left\{\frac { 1 } { m _ { B } \beta } \left[\frac{1}{(1+\beta p)}\right.\right. \\
\left.-\frac{2 \tanh ^{-1}\left((2 \beta p+1) / \sqrt{4 m_{B} \beta+1}\right)}{\sqrt{4 m_{B} \beta+1}}\right] \\
+\frac{1}{m_{B} \beta^{2}}[\ln (1+\beta p) \\
-\frac{\tanh ^{-1}\left((2 \beta p+1) / \sqrt{4 m_{B} \beta+1}\right)}{\sqrt{4 m_{B} \beta+1}} \\
\left.\left.\quad-\frac{1}{2} \ln \left(\beta^{2} p^{2}+\beta p-m_{B} \beta\right)\right]\right\} .
\end{array}
$$

The probability for the process to occur is given by $|M|^{2}$. Now we calculate the scattering amplitude in the presence of both minimal length and maximal momentum. In this case, $p \rightarrow$ $p\left(1-\beta p+2 \beta^{2} p^{2}\right)$, and we have

$$
\begin{aligned}
& M \\
& =\int_{-p_{p l}}^{+p_{p l}}-i g^{2} \frac{d p}{\left[\left(p\left(1-\beta p+2 \beta^{2} p^{2}\right)\right)^{2}-m_{B}^{2}\right]\left(1-\beta p+2 \beta^{2} p^{2}\right)}
\end{aligned}
$$

which can be simplified to find

$$
M=\int_{-p_{p l}}^{+p_{p l}}-i g^{2} \frac{d p}{p\left(1-\beta p+2 \beta^{2} p^{2}\right)^{3}-m_{B}\left(1-\beta p+2 \beta^{2} p^{2}\right)} .
$$

By solving this integral, we find the following result for scattering amplitude in the presence of both minimal length and maximal momentum:

$$
\begin{aligned}
& M=-i g^{2} \frac{1}{2 \beta^{2} m_{B}^{2}} \\
& \times\left\{\sum_{R} \frac{(1+R)^{2}}{3 R^{2}+2 R+7} \ln \left(4 \beta p_{p l}-1-R\right)\right. \\
& -\sum_{R} \frac{(1+R)^{2}}{3 R^{2}+2 R+7} \ln \left(-4 \beta p_{p l}-1-R\right) \\
& +\frac{1}{2} \ln \frac{\left(4 \beta p_{p l}+1\right)^{2}+7}{\left(4 \beta p_{p l}-1\right)^{2}+7} \\
& \left.-\frac{1}{\sqrt{7}}\left(\tan ^{-1} \frac{4 \beta p_{p l}-1}{\sqrt{7}}+\tan ^{-1} \frac{4 \beta p_{p l}+1}{\sqrt{7}}\right)\right\} \\
& +\frac{8 i g^{2}}{7 \beta m_{B}}\left\{\frac{4 \beta p_{p l}-1}{\left(4 \beta p_{p l}-1\right)^{2}+7}+\frac{4 \beta p_{p l}+1}{\left(4 \beta p_{p l}+1\right)^{2}+7}\right. \\
& +\frac{1}{\sqrt{7}}\left(\tan ^{-1} \frac{4 \beta p_{p l}-1}{\sqrt{7}}\right. \\
& \left.\left.+\tan ^{-1} \frac{4 \beta p_{p l}+1}{\sqrt{7}}\right)\right\}
\end{aligned}
$$

where by definition $R$ is the root of $2 z^{2}+7 z+7-32 \beta^{32} m_{B}=0$. Now the probability for the process to occur in the presence of these natural cutoffs is given by $|M|^{2}$.

\section{Summary}

In this paper, we have constructed a quantum theory of free fermion fields based on the deformed Heisenberg algebra that contains both a minimal measurable length and a maximal momentum for test particles. Our strategy was to respect the supersymmetry in this context as a guiding principle. This is because string theory contains this symmetry, and it provides a useful tool to understand physics in ultraviolet regime. Due to existence of natural cutoffs as minimal length and maximal momentum, the supersymmetry is deformed in this context. From the fermionic part of the system, we proposed an action of fermionic field based on the GUP in a spacetime with arbitrary number of dimensions. We have constructed the deformed supersymmetric algebra in this context. Finally, we have computed the scattering amplitude for pair annihilation 
to see how these important quantities can be calculated in the presence of natural cutoffs as minimal length and maximal momentum.

\section{References}

[1] A. Bilal, "Introduction to supersymmetry," http://arxiv.org/abs/ hep-th/0101055.

[2] S. Hossenfelder, "Minimal length scale scenarios for quantum gravity," Living Reviews in Relativity, vol. 16, p. 2, 2013.

[3] A. Kempf, "Quantum field theory with nonzero minimal uncertainties in positions and momenta," http://arxiv.org/abs/hepth/9405067.

[4] A. Kempf and G. Mangano, "Minimal length uncertainty relation and ultraviolet regularization," Physical Review D, vol. 55, no. 12, pp. 7909-7920, 1997.

[5] S. Hossenfelder, "Interpretation of quantum field theories with a minimal length scale," Physical Review D, vol. 73, no. 10, Article ID 105013, 9 pages, 2006.

[6] G. Amelino-Camelia, "Relativity in space-times with shortdistance structure governed by an observer-independent (Planckian) length scale," International Journal of Modern Physics D, vol. 11, no. 1, pp. 35-59, 2002.

[7] G. Amelino-Camelia, "Relativity: special treatment," Nature, vol. 418, no. 6893, pp. 34-35, 2002.

[8] G. Amelino-Camelia, "Doubly-special relativity: first results and key open problems," International Journal of Modern Physics D, vol. 11, no. 10, pp. 1643-1669, 2002.

[9] R. Aloisio, A. Galante, A. F. Grillo, E. Luzio, and F. Méndez, "Approaching space time through velocity in doubly special relativity," Physical Review D, vol. 70, no. 12, Article ID 125012, 9 pages, 2004.

[10] G. Amelino-Camelia, J. Kowalski-Glikman, G. Mandanici, and A. Procaccini, "Phenomenology of doubly special relativity," International Journal of Modern Physics A, vol. 20, no. 26, pp. 6007-6037, 2005.

[11] K. Nozari and A. Etemadi, "Minimal length, maximal momentum and Hilbert space representation of quantum mechanics," Physical Review D, vol. 85, Article ID 104029, 12 pages, 2012.

[12] P. Pedram, K. Nozari, and S. H. Taheri, "The effects of minimal length and maximal momentum on the transition rate of ultra cold neutrons in gravitational field," Journal of High Energy Physics, vol. 2011, no. 3, article 93, 2011.

[13] G. Amelino-Camelia, "Gravity-wave interferometers as probes of a low-energy effective quantum gravity," Physical Review D, vol. 62, no. 2, Article ID 024015, 18 pages, 2000.

[14] G. Amelino-Camelia, D. Benedetti, F. D’Andrea, and A. Procaccini, "Comparison of relativity theories with observerindependent scales of both velocity and length/mass," Classical and Quantum Gravity, vol. 20, no. 24, pp. 5353-5370, 2003.

[15] A. F. Ali, S. Das, and E. C. Vagenas, "Proposal for testing quantum gravity in the lab," Physical Review D, vol. 84, no. 4, Article ID 044013, 10 pages, 2011.

[16] G. Amelino-Camelia, "Quantum spacetime phenomenology," Living Reviews in Relativity, vol. 16, p. 5, 2013.

[17] A. Borowiec, J. Lukierski, and V. N. Tolstoy, "Basic twist quantization of $\operatorname{osp}(1 \mid 2)$ and $\kappa$-deformation of $D=1$ superconformal mechanics," Modern Physics Letters A, vol. 18, no. 17, pp. 11571169, 2003.
[18] H. Hinrichsen and A. Kempf, "Maximal localization in the presence of minimal uncertainties in positions and in momenta," Journal of Mathematical Physics, vol. 37, no. 5, pp. 2121-2137, 1996.

[19] Y. Shibusa, "Supersymmetric field theory based on generalized uncertainty principle," International Journal of Modern Physics A, vol. 22, no. 29, pp. 5279-5286, 2007.

[20] D. McMahon, Quantum Field Theory Demystified, McGrawHill, 2008. 

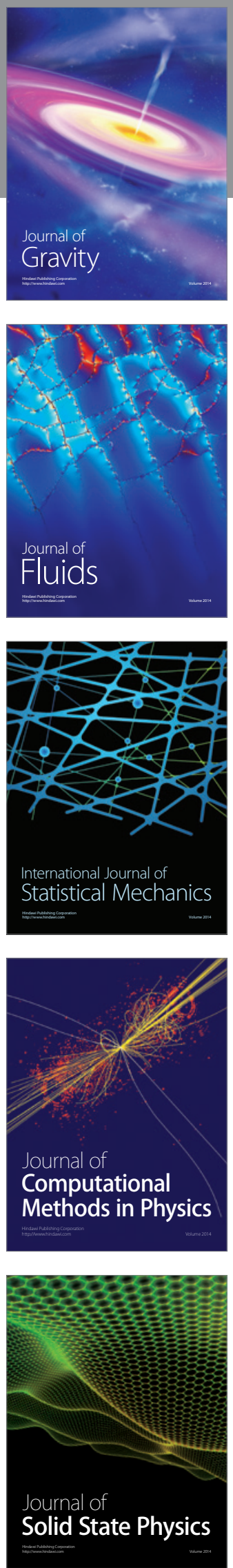

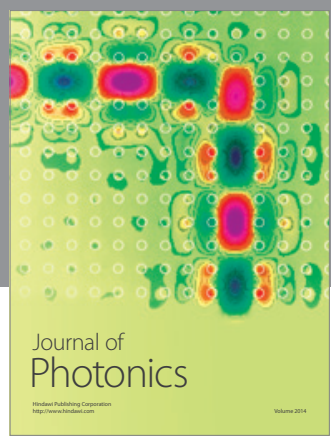

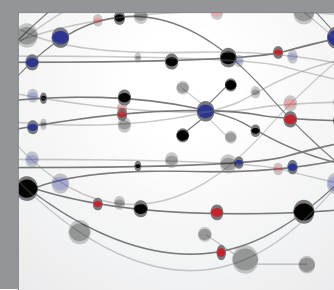

The Scientific World Journal

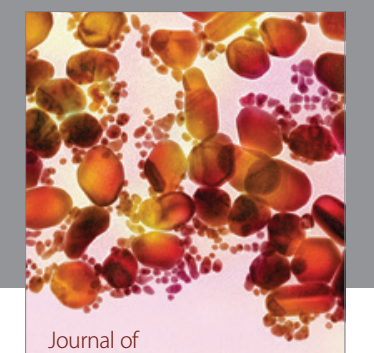

Soft Matter
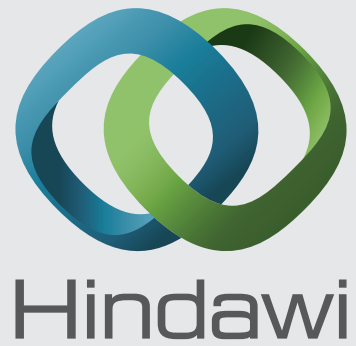

Submit your manuscripts at

http://www.hindawi.com
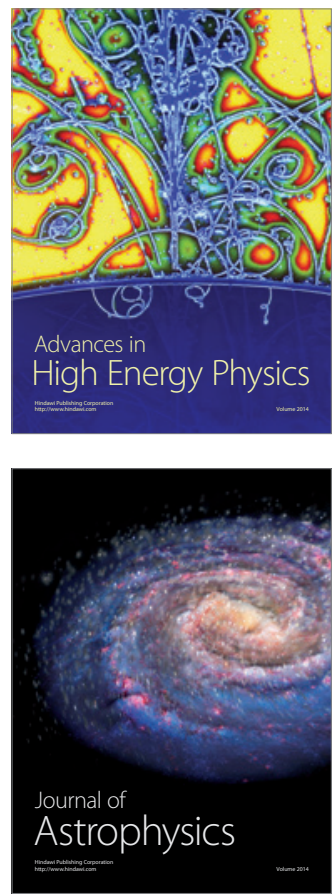
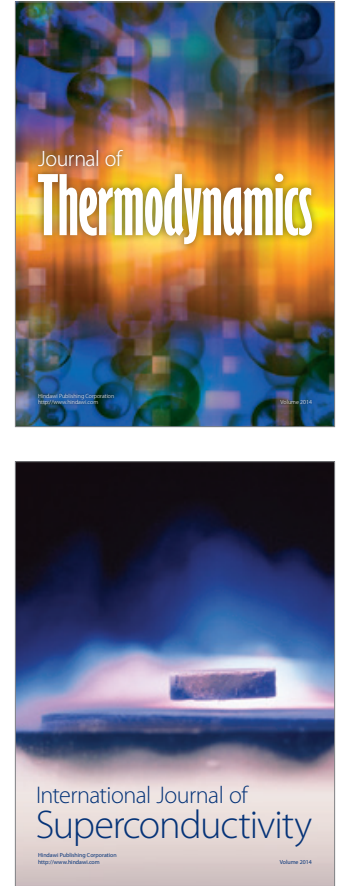
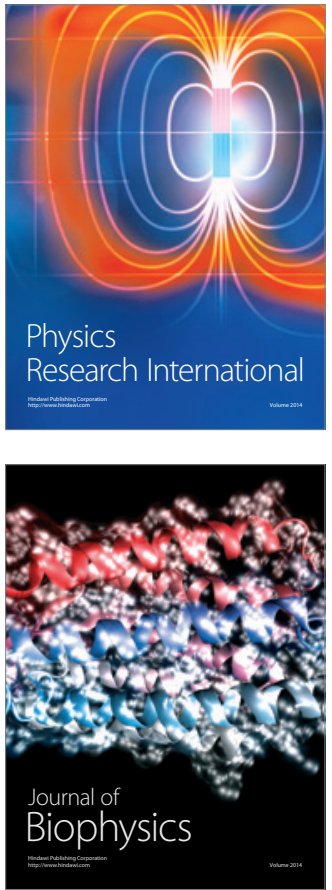
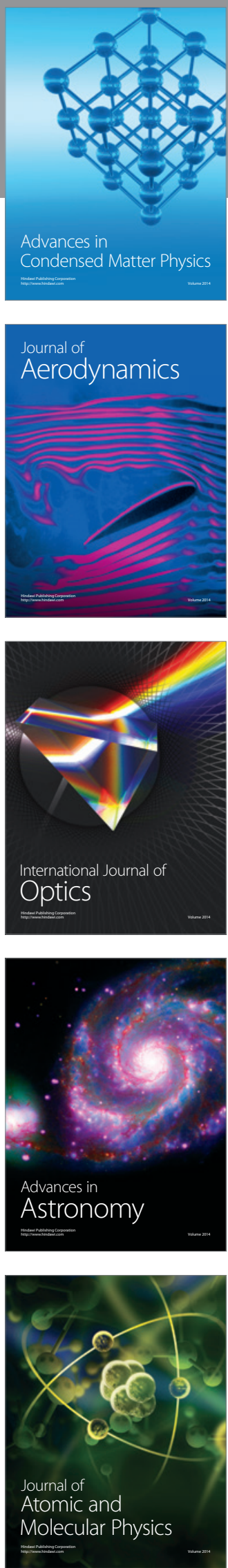